\title{
Primer registro fósil de Parasitengona (Acari: Prostigmata) del ámbar mexicano, con una descripción de un nuevo género y especie para la familia Johnstonianidae Thor, 1935
}

First fossil record of Parasitengona (Acari: Prostigmata) from Mexican amber, with description of a new genus and species for family Johnstonianidae Thor, 1935

Gerardo Rivas $^{1}$, Francisco J.Vega ${ }^{2, *}$

${ }^{1}$ Facultad de Ciencias, Universidad Nacional Autónoma de México, Ciudad Universitaria, Coyoacán, 04510, CDMX, México.

${ }^{2}$ Instituto de Geología, Universidad Nacional Autónoma de México, Ciudad Universitaria, Coyoacán, CDMX, 04510, México.

* Autor para correspondencia: (F.J. Vega) vegver@unam.mx

\section{RESUMEN}

Se describe Palaeodiplothrombidium microscutum gen, et sp. nov. a partir de un adulto y una deutoninfa preservados en ámbar del Mioceno inferior de Chiapas, México. Este taxón nuevo de ácaro es el primer registro de la Cohorte Parasitengona (Acari: Trombidiformes: Prostigmata) para un yacimiento de ámbar dentro de México y también el primer registro fósil de la familia Johnstonianidae a nivel mundial.

Palabras clave: Ácaro terciopelo, ámbar, Mioceno temprano, Chiapas, México.

\section{Cómo citar este artículo:}

Rivas, G., Vega, FJ., 2021, Primer registro fósil de Parasitengona (Acari: Prostigmata) del ámbar mexicano, con una descripción de un nuevo género y especie para la familia Johnstonianidae Thor, 1935: Boletín de la Sociedad Geológica Mexicana, 73 (3), Al70521. http://dx.doi.org/10.18268/ BSGM2021v73n3a170521.

Manuscrito recibido: 20 de Noviembre de 2020

Manuscrito corregido: 25 de Diciembre de 2020 Manuscrito aceptado: 11 de Enero de 2021

La revisión por pares es responsabilidad de la Universidad Nacional Autónoma de México.

Este es un artículo Open Access bajo la licencia CC $1-N C$ SA (https://creativecommons.org/licenses/by-nc-sa/4.0/)

\section{ABSTRACT}

Palaeodiplothrombidium microscutum gen. et sp. nov. is described based on one adult and one deutonymph preserved in early Miocene amber from Chiapas México. This new taxon is the first record for the Parasitengona Cohort (Acari: Trombidiformes: Prostigmata) for the Chiapas amber and is also the first fossil record for the Fohnstonianidae Family, worldwide.

Keywords: Velvet mite, amber, early Miocene, Ghiapas, Mexico. 


\section{Introducción}

Parasitengona es una cohorte de ácaros prostigmados, cuya característica principal es tener en su ciclo de vida una fase larvaria parásita mientras que el adulto y la deutoninfa son activos depredadores de vida libre (Walter et al., 2009; Wohltmann et al., 2006). Dentro de este linaje se encuentra la subcohorte Hydrachnidiae, donde se incluyen las cerca de 5000 especies de ácaros acuáticos y también las subcohortes Erythraiae y Trombidiae, ambos con una radiación adaptativa principalmente terrestre (Wohltmann et al., 2006).

En Trombidiae se incluye, entre otras, a la familia Trombiculidae, cuyas larvas parasitan a vertebrados incluyendo al hombre, desconociéndose incluso para muchas especies la fase adulta. Otras familias dentro de Trombidiae son Trombidiidae, Neothmbidiidae, Microtrombidiidae, Trombellidae y Johnstonianidae, cuyas especies en general se conocen comúnmente como ácaros terciopelo (Wohltmann et al., 2006), este es uno de los pocos grupos de Acari que pueden ser visibles relativamente a simple vista y cuyo nombre deriva de la gran cantidad de sedas que cubre toda la superficie del idiosoma.

El registro fósil de los Parasitengona terrestres (Trombidiae) ha sido favorecido en los yacimientos de ámbar, siendo relevantes las localidades del Báltico, de donde se han descrito especies de las familias Microtrombidiidae, Tanaupodidae y Trombidiidae (Konikiewicz et al., 2016; Mąkol et al., 2018; Dunlop et al., 2020) y recientemente en el ámbar Cretácico de Myanmar, con la descripción de especies nuevas de Trombellidae (Konikiewicz y Mąkol, 2018).

Hasta la fecha, se han registrado 12 especies de ácaros en el ámbar mexicano (Norton y Poinar, 1993; Rivas et al., 2016), de las cuales solo una pertenece al taxón Prostigmata, pero no a Parasitengona, por lo que la presente contribución tiene como objetivo brindar el primer registro del clado Parasitengona para el ámbar de México, así como la descripción de un género y especie nueva para la familia Johnstonianidae.

\section{Localidad y estratigrafía}

La muestra estudiada procede de las minas de Campo La Granja, ubicadas al norte de Simojovel, Chiapas, y representan la fase inicial de la generación y depósito de ámbar, durante el Mioceno temprano (Serrano-Sánchez et al., 2015). El paleoambiente interpretado para el ámbar estratificado de las minas de Campo La Granja, corresponde a un estero cercano a la costa ancestral del Golfo de México.

La microfauna (principalmente crustáceos estuarinos) que contienen las piezas de Campo La Granja, incluye copépodos, ostrácodos, larvas parásitas de isópodos, tanaidáceos, anfipodos, camarones y cangrejos estuarinos. Para ubicación, estratigrafía e interpretación ambiental, consultar Serrano-Sánchez et al. (2015).

\section{Métodos}

Las fotos se tomaron usando campo claro. Todas las medidas están en micrómetros. Las abreviaturas utilizadas en la descripción para las medidas e imágenes fueron: $B f=$ basifémur, $b s=$ basidonto, $C x=$ coxa,$e y=$ ojos, $G e=$ genua, $o d=$ odonto, $T a$ $=\operatorname{tarso}, T i=$ tibia, $T f=$ telofémur y $T r=$ trocánter. Guando no fue posible distinguir los límites en los artejos de los apéndices, se registró la medición en conjunto, ejemplo: $T f-T r$ significa el largo de la unión del telofémur más el basifémur más el trocánter.

\section{Paleontología sistemática}

Subclase Acari Sundevall, 1833

Superorden Acariformes Zakhvatkin, 1952

Orden Trombidiformes Reuter, 1909

Suborden Prostigmata Kramer, 1877

Cohorte Parasitengona Oudemans, 1909

Familia Johnstonianidae Thor, 1935

Género Palaeodiplothrombidium gen. nov. 
Diagnosis: Estados postlarvales activos: Ácaros de tamaño mediano $(<1000 \mu)$, idiosoma cubierto densamente con sedas, contorno del idiosoma no ovoidal. Scutum triangular en la parte anterior y elongado en la posterior, de longitud pequeña con relación al tamaño del idioma, (largo del scutum es cerca de la novena parte del largo del idiosoma). Tarso del pedipalpo perpendincular a la tibia. Odonto de la tibia grueso y basidonto de tipo unidentado.

\section{Larva: desconocida.}

Etimología: de la raíz griega palaeo "antiguo", por ser el único taxón fósil dentro de la familia y en alusión también a su parecido y cercana relación al actual género Diplothrombium.

Palaeodiplothrombidium microscutum gen. et sp. nov.

Diagnosis: Misma que la del género.

Material tipo: Holotipo: Un adulto (IHNFG5989) y paratipo, una deutoninfa (IHNFG-5990). La pieza se encuentra depositada en el Museo de Paleontología "Eliseo Palacios Aguilera", Secretaría de Medio Ambiente e Historia Natural, Estado de Chiapas, México.

Descripción: Adulto: contorno del idiosoma tipo trapezoidal, aunque con el extremo posterior redondeado y ligeramente constreñido a la altura de las patas III (Figuras 1A a 1C), largo del idiosoma $870 \mu$ y ancho máximo $605 \mu$. El primer par de patas más largo que el resto. Sedas espiniformes. Scutum triangular en la parte anterior y elongado en la posterior (Figura 2A). Crista metópica delgada y alargada con dos áreas de redondeadas presumiblemente de inserción de sedas (Figuras 2A y 2B). En la porción medía del idiosoma, el ejemplar se encuentra un poco maltratado por lo que da una apariencia asimétrica sobre todo al tratar de ubicar las áreas genital y anal (Figura 1C), en el caso de la primera (largo aproximado de $100 \mu$ ) débilmente distinguible a diferencia de la segunda. La quetotaxia de los pedilpalpos no es visible, a excepción del tarso donde se distinguen alrededor de cinco sedas largas que podrían ser eupatidios y en la tibia el odonto y el basidonto, este último mucho más delgado que el primero, tarso perpendicular a la tibia (Figuras 2C y 2D). Largo de los artejos del pedipalpo (Ta $23 \mu$, Ti $75 \mu$, Ge $32 \mu$, Fe $56 \mu$ ); largo del odonto $(19 \mu)$ y del basidonto $(12 \mu)$, distancia entre el odonto y basidonto $(11 \mu)$. Largo de los artejos de las patas: Leg I (Ta $160 \mu$, Ti $120 \mu$, Ge $110 \mu$, Tf $75 \mu$, Bf $25 \mu$, Tr $67 \mu$, Cx $120 \mu$ ); leg II (Ta -, Ti $90 \mu$, Ge $66 \mu$, Tf $68 \mu$, Bf-Tr $75 \mu$, Cx 97 н); leg III (Ta-Tr $340 \mu$, Cx $95 \mu$ ); leg IV (Ta $170 \mu$, Ti $90 \mu$, Ge $85 \mu$, Tf-Tr $150 \mu$, Cx $120 \mu$ ). Largo de las sedas del idiosoma $(10-15 \mu)$.

Deutoninfa: idiosoma cubierto por sedas, largo $440 \mu$ y ancho $230 \mu$ (Figura 1D). Ejemplar maltratado con burbujas y opacidad que dificultan su observación, de igual manera las patas se encuentran dobladas y no es posible la medición de sus artejos a excepción del tarso de la pata I: $71 \mu$. Medidas del pedipalpo: Ta $18 \mu$, Ti $35 \mu$, Ge $36 \mu$ y Fe $48 \mu$.

Etimología: De las raíces griegas "micro" pequeño y "scutum" escudo, en alusión a lo pequeño del scutum con relación a la magnitud del idiosoma.

Observaciones: Palaeodiplothrombidium gen. nov. se distingue entre los otros géneros de la familia Fohnstonianidae Thor, 1935 por tener el basidonto unidentado (no bidentado como en fohnstoniana George, 1909) y el scutum elongado (no triangular como en Newellia André, 1962). La forma elongada del scutum la comparte con los géneros Centrotrombidium Kramer, 1896, Diplothrombium Berlese, 1910 y Paraplothrombium Robaux, 1968; aunque la constricción anterior en la crista metópica característica en ese último no se visualiza en Palaeodiplothrombidium gen. nov., además de que el nuevo taxón presenta dos pares de áreas de inserción de las trichobotria en la base de la crista metópica a diferencia de un solo par tal como se aprecia en la descripción de Paraplothrombidium.

La crista metópica de Palaeodiplothrombidium microscutum gen. et sp. nov. es semejante a la de las especies europeas Diplothrombium carpaticum (Štorkan, 1938), D. longipalpe (Berlese, 1887), así como a $D$. monoense y D. micidium descritas para Norteamérica por Newell (1957), sin embargo, el largo total del scutum (escudo que contiene a la crista metópica) en la especie nueva es prácticamente la cuarta parte respecto al largo promedio de las otras tres especies mencionadas. Situación semejante se presenta con 

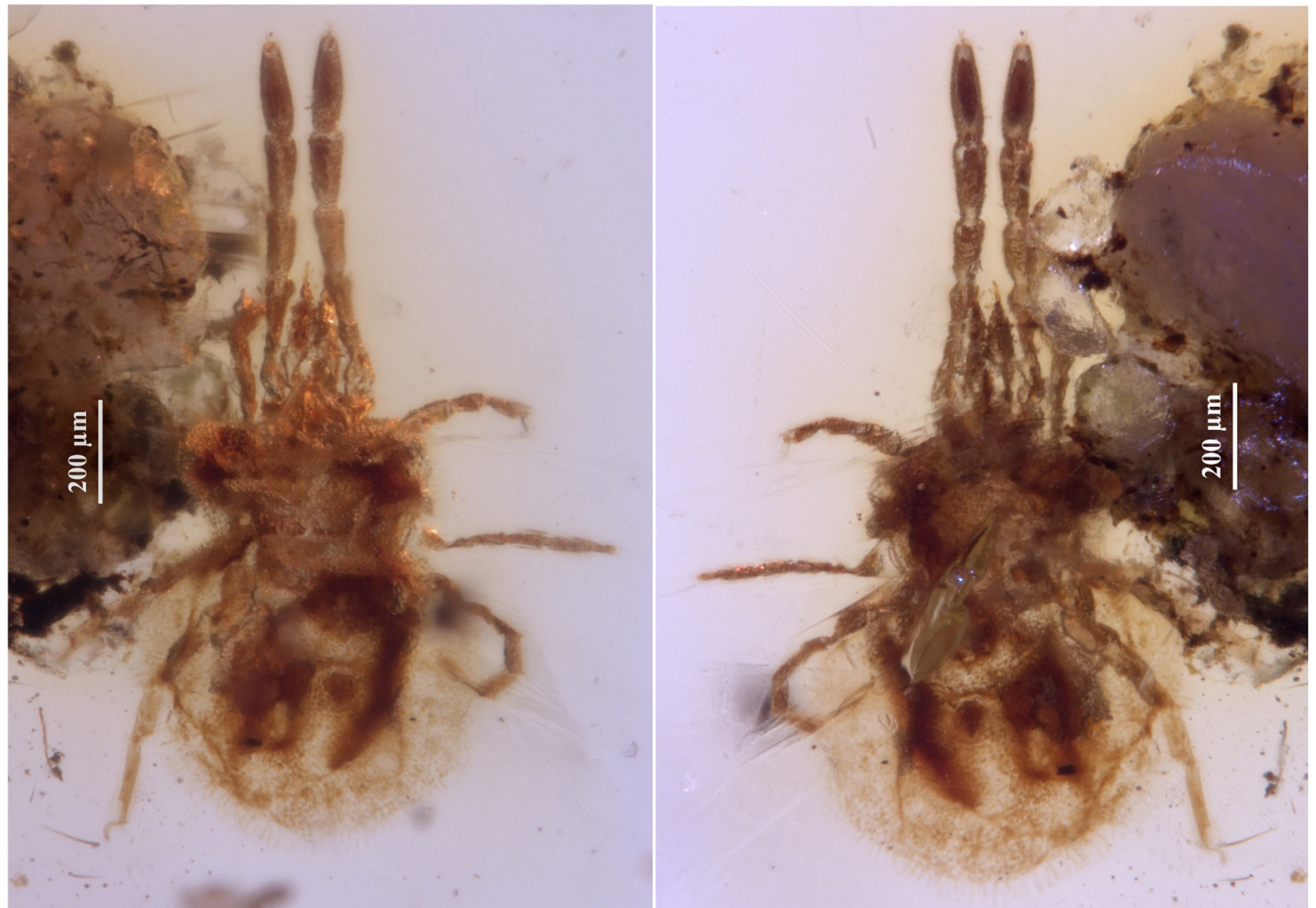

B

.

A

C

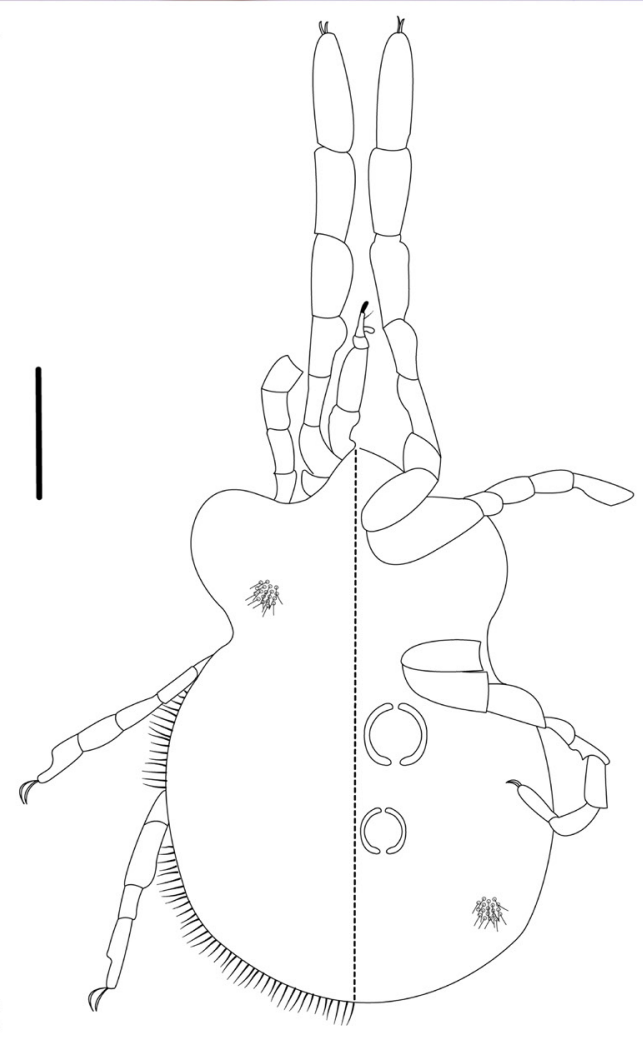

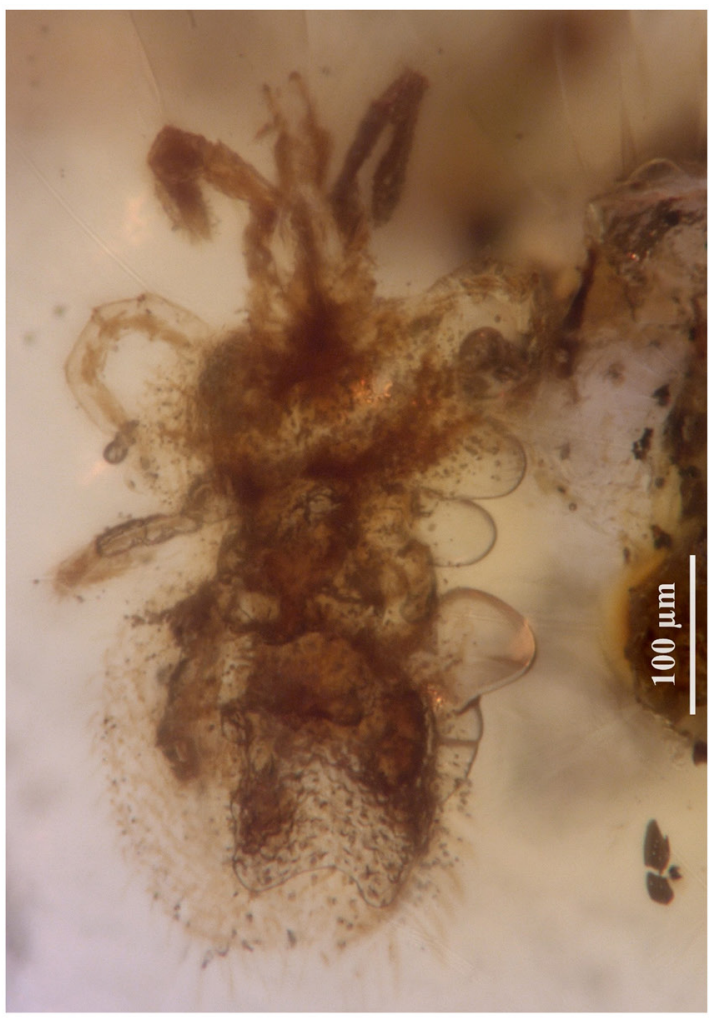

Figura 1 Palaeodiplothrombidium microscutum gen. et sp. nov. A, B, vista dorsal del ejemplar adulto. C. Vista dorsal (derecha) y ventral (izquierda) del adulto (escala $=200 \mu \mathrm{m}$ ). D. Vista dorsal de la deutoninfa. 

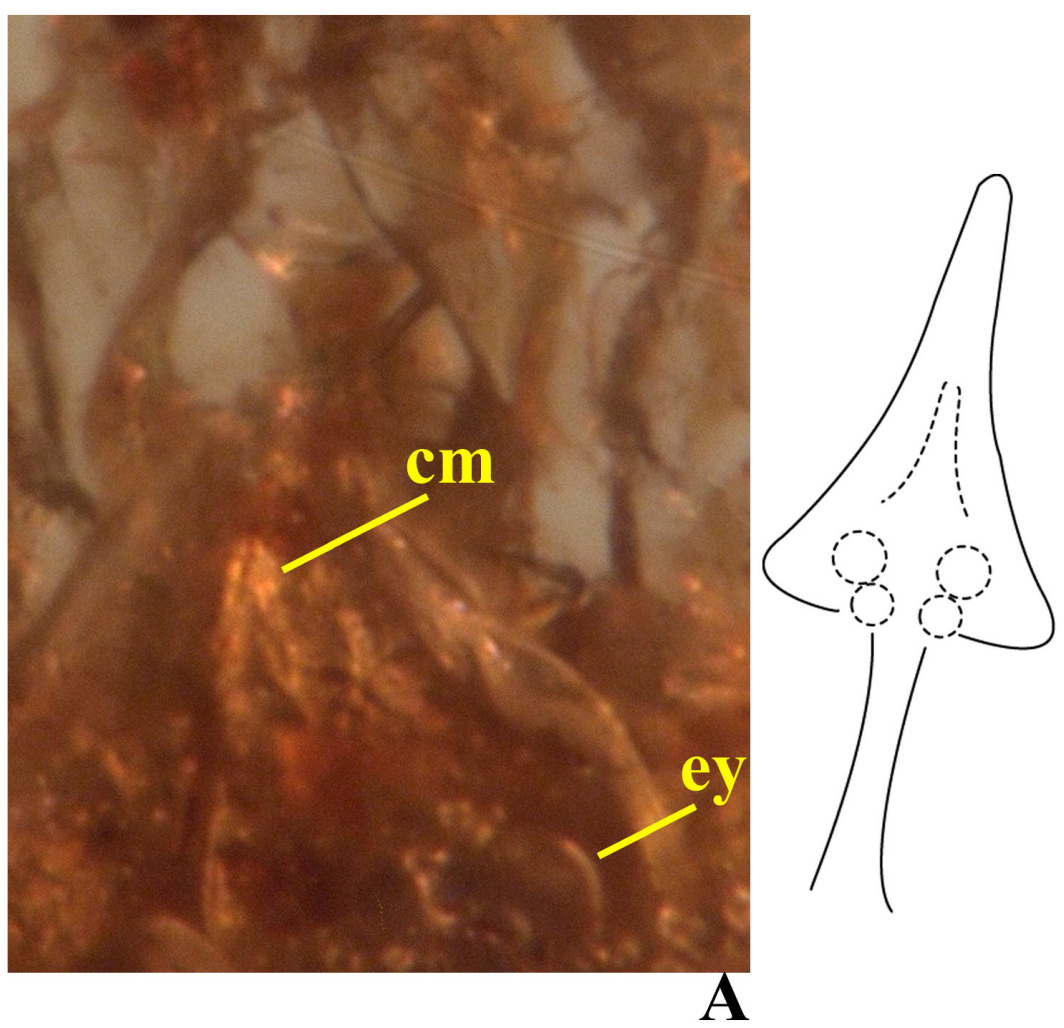

A
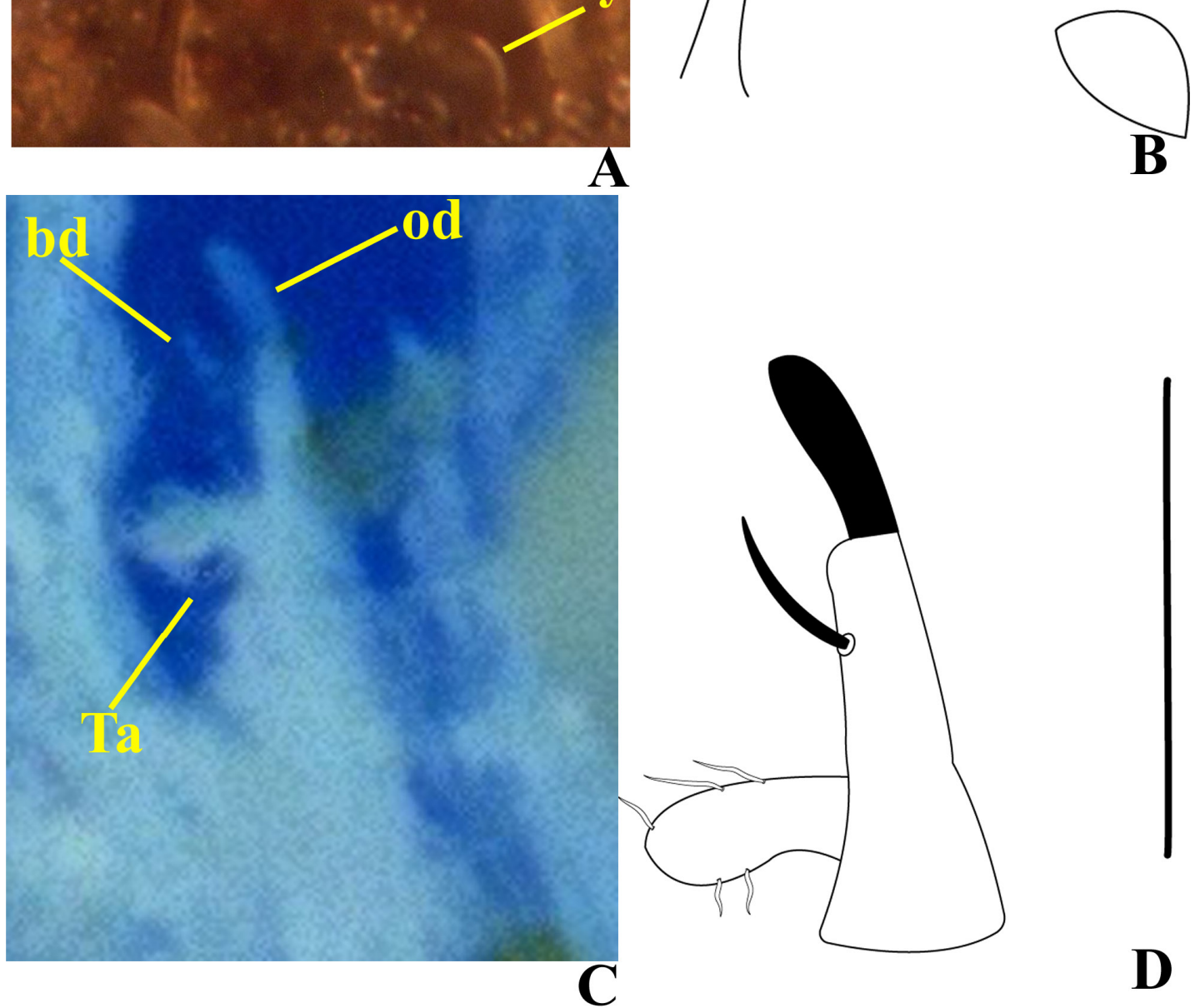

Figura 2 Palaeodiplothrombidium microscutum sp. nov. A y B, porción anteromedio dorsal del idiosoma: crista metópica (cm), ojos (ey). C y D, porción distal del pedipalpo: Tarso (Ta), odontus (od) y basidonto (bs) (escala $=50 \mu \mathrm{m}$ ). 
otras especies descritas por Newell (1957), pero del género Centrotrombidium: C. approximatum, $\mathrm{C}$ distans y C. hadroseta, sin embargo, estas tres no presentan la proyección posterior en la crista metópica, a diferencia de Palaeodiplothrombidium microscutum gen. et sp. nov.

\section{Discusión}

Los Parasitengona "terrestres" (subcohortes Erythraiae y Trombidiae) han denotado relativo interés en su estudio debido a su gran tamaño y su apariencia estética por la gran cantidad de sedas en su idiosoma que dan la apariencia aterciopelada. Estos dos linajes de ácaros prostigmados incluyen entre 7 y 9 mil especies nominales (Wohltmann et al., 2004), lo que refleja una riqueza específica considerable, pero a la vez compleja.

El estado actual de la taxonomía es bastante deficiente en la mayoría de las familias de afinidad terrestre de Parasitengona, principalmente porque no en todas las especies se ha encontrado la larva y/o los estados postlarvales activos (deutoninfa y adulto), además no para todos los géneros existen revisiones exhaustivas, por lo que es necesario incrementar el conocimiento taxonómico de estos grupos.

La quetotaxia es un referente importante para la taxonomía y filogenia de los ácaros, y especialmente en Parasitengona resulta importante con las larvas. En este sentido, la limitante en ejemplares fósiles de no contar con esta valiosa información representa un problema severo de conciliación con los taxones actuales.

$\mathrm{Al}$ respecto, el taxón nuevo que se propone en el presente estudio pudiera eventualmente ser incluido dentro del género Diplothrombidium, sin embargo, el largo y ancho máximo del scutum, y las dimensiones de los segmentos del pedipalpo de la nueva especie, son evidentemente más pequeñas que el promedio para las especies descritas de Diplothrombidium; por ejemplo, el intervalo del largo del scutum oscila entre $345 \mu$ y $710 \mu$, mientras en nuestra especie mide tan solo $100 \mu$ (al menos la cuarta parte), y si bien el idiosoma de Palaeodiplothrombidium gen. nov. puede ser considerado "mediano", el largo de este (casi $900 \mu$ ) no dista mucho del intervalo para del género Diplothrombium (1000-1500 p), no al menos dentro del mismo orden de magnitud que para el caso del scutum.

Situación similar ocurre con la comparación entre Palaeodiplothrombidium gen. nov. respecto a Centrotrombium, aunque dada la terminación no tan puntiaguda del extremo anterior del scutum del género nuevo y la forma no ovoide del idiosoma, hace que tenga una relativa mayor afinidad con Diplothrombium. El contorno del idiosoma del genéro nuevo que se propone es incluso similar al que se presenta en la familia Trombiculidae lo cual consideramos como una convergencia dentro de la amplia diversidad de los Parasitengona terrestres. Dada la falta de visualización de la posible presencia de las sedas en los pedipalpos y a lo largo de la crista metópica en nuestro ejemplar fósil, resulta aún más subjetiva la asignación dentro alguno de los géneros descritos dentro de Johnstonianidae, por tal motivo se propone la formación de un género nuevo, en especial para lo que representaría a nivel mundial el primer registro fósil dentro de la familia.

Johnstonianidae, al igual que otras familias de la subcohorte Trombidiae, pueden habitar en biotopos hídricos (Wohltmann et al., 2006). Las larvas generalmente son parásitas de insectos semiacuáticos, es decir que presentan al menos una fase de su desarrollo dentro del agua, tales como coleópteros, tricópteros y dípteros de las familias Ceratopogonidae, Dixidae, Pediciidae y Tipulidae (Wohltmann et al., 2004; Felska et al., 2018)

\section{Agradecimientos}

Nuestra gratitud para los Dres. Joanna Makol, Ignacio Vázquez Rojas, Ricardo Paredes, Grzegorz Gabrys y Andreas Wohltmann por su invaluable apoyo con referencias bibliográficas. Nuestro agradecimiento a Mauricio Ramírez por su valiosa ayuda en la elaboración de los dibujos. 


\section{Referencias}

André, M., 1962, Acariens Thrombidions (adultes) de l'Angola (2éme note): Publicaçōes Culturais Companhia de Diamantes de Angola (Diamang), 60, 57-112.

Berlese, A., 1887, Acari, Myriapoda et Scorpioneshucusque in Italia reperta: Padova 1882-1893. Padova 1893. Fascicolo 42(2). https://doi.org/10.5962/bhl.title.69269

Berlese, A., 1910, Brevidiagnosi di generi e specienuovi di Acari: Redia, 6, 346-388.

Dunlop, J.A., Penney, D., Jekel, D., 2020, A summary list of fossil spiders and their relatives. In, World Spider Catalog. Natural History Museum Bern. Disponible en http:/ / wsc.nmbe.ch, version 20.5.

Felska, M., Wohltmann, A., Mąkol, J., 2018, A synopsis of host-parasite associations between Trombidioidea (Trombidiformes: Prostigmata, Parasitengona) and arthropod hosts: Systematic \& Applied Acarology, 23, 1375-1479. https://doi.org/10.11158/ saa.23.7.14

George, C.F., 1909, Some British earth mites: Naturalist (London), 281-282.

Konikiewicz, M., Mąkol, J., 2018, Insight into fossil fauna of terrestrial Parasitengona mites (Trombidiformes: Prostigmata) - The first representatives of Erythraeina Welbourn, 1991 and Trombidiina Welbourn, 1991 in Burmese amber: Cretaceous Research, 89, 60-74. https://doi.org/10.1016/j. cretres.2018.02.017

Konikiewicz, M., Sontag, E., Mąkol, J., 2016, The first description of a microtrombidiid mite (Actinotrichida: Prostigmata, Microtrombidiidae) from Baltic amber, with notes on related extant genera and species: Palaeontologische Zeitschrift, 90, 493-501. https://doi.org/10.1007/ s12542-016-0311-y

Kramer, P., 1877, Grundzüge zur Systematik der Milben: Archiv für Naturgeschichte, Neue Folge, Berlin, 43, 215-247.
Kramer, P., 1896, Neue Acarididen von der Insel Borkum: Zoologischer Anzeiger, 19, 444-448. Mąkol, J., Konikiewicz, M., Klug, B., 2018, Next ones to fill the gap-first tanaupodids (Trombidiformes: Prostigmata, Tanaupodidae) from Baltic amber with notes on extant genera and species: Systematic and Applied Acarology, 23(11), 2129-2146. https://doi.org/10.11158/saa.23.11.6

Newell, I.M., 1957, Studies on the Johnstonianidae (Acari, Parasitengona): Pacific Science, 11(4), 396-466.

Norton, R.A., Poinar, G.O., 1993, Reassessment and new records of oribatid mite fossils from Tertiary Neotropical amber: Acarologia, 34(1), 57-68.

Oudemans,A.C., 1909 , A c a rologische Aanteekeningen XXIX: Entomologische Berichten, 3, 14-24.

Oudemans, A.C., 1937, Kritsch historisch Overzicht der acarologie. Vol. 3, 1805-1850: Leiden, E. J. Brill, 3379 p.

Reuter, E., 1909, Zur Morphologie und Ontogenie der Acariden mit besonderer Berücksichtigung von Pediculopsisgraminum (E. Reuter): Acta Societatis Scientiarum Fennicae, 36, 1-288. https://doi. org/10.5962/bhl.title.4705

Rivas, G., Serrano-Sánchez, L., Vega, FJ., 2016, First record of Procaeculus (Acari: Caeculidae) in Miocene amber from Chiapas, Mexico: Boletín de la Sociedad Geológica Mexicana, 68(1), 87-92. http://dx.doi.org/10.18268/ BSGM2016v68n1a10

Robaux, P., 1968, Thrombidiidae d'Amérique du sud I - Tanaupodidae, Johnstonianinae, Thrombellini. (Acarina-Thrombidiidae): Acarologia, 10(3), 450-466.

Robaux, P., 1974, Recherches sur le développement et la biologie des acariens 'Thrombidiidae': Mémoires du Muséumnational d'histoirenaturelle Paris (n.s.). Série A, Zoologie, 85, 1-186.

Serrano-Sánchez, M.L., Hegna, T.A., Schaaf, P., Pérez, L., Centeno-García, E., Vega, FJ., 
2015, The aquatic and semiaquatic biota in Miocene amber from the Campo La Granja mine (Chiapas, Mexico): Paleoenvironmental implications: Journal of South American Earth Sciences, 62, 243-256. https://doi. org/10.1016/j.jsames.2015.06.007

Štorkan, J., 1938, Fohnstoniana carpatica n. sp. (Thrombidiidae, Acar.): Zoologischer Anzeiger, 5(6), 110-114.

Sundevall, J.C., 1833, Conspectus Arachnidum. Londini Gothorum, 1-39.

Thor, S., 1935, Übersicht und Einteilung der Familie Trombidiidae W.E. Leach, 1814 in Unterfamilien: Zoologischer Anzeiger, 109, 107-112.

Walter, D.E., Lindquist, E.E., Smith, I.M., Cook, D.R., Krantz, G.W., 2009, Order trombidiformes in Krantz, G.W., Walter, D.E. (eds.), A manual of Acarology, Lubbock Texas, Texas Tech University Press, 64-82.

Wohltmann, A., 2001, The evolution of life histories in Parasitengona (Acari: Prostigmata): Acarologia, 41, 145-204.

Wohltmann, A., Gabryś, G., Mąkol, J., 2006, Acari: Terrestrial Parasitengona inhabiting transient biotopes. en Gerecke, R. (ed.), Süßwasserfauna von Mitteleuropa 7(2-1), Chelicerata, Araneae, Acari I. Süßwasserfauna von Mitteleuropa: Berlin, Springer, 158-240. https://doi. org/10.1007/978-3-662-55958-1_6

Wohltmann, A., Mąkol, J., Gabryś, G., 2004, A revision of European Johnstonianinae Thor, 1935 (Acari: Prostigmata: Parasitengona: Trombidioidea): Annales Zoologici, 54(3), 595-630. https://doi. org/10.3161/0003454043598140

Zakhvatkin, A.A., 1952, Subdivision of the mites (Acarina) into orders and the position of these in the system of Chelicerata: Parazitologicheskii Sbornik Zoologicheskii Institut Akademii Nauk SSSR, 12, 5-46. 